\title{
Superconducting qubits can be coupled and addressed as trapped ions
}

\author{
Yu-xi Liu, ${ }^{1,2}$ L. F. Wei, ${ }^{1,2}$ J. R. Johansson, ${ }^{2}$ J. S. Tsai, ${ }^{1,2,3}$ and Franco Nori ${ }^{1,2,4}$ \\ ${ }^{1}$ CREST, Japan Science and Technology Agency (JST), Kawaguchi, Saitama 332-0012, Japan \\ ${ }^{2}$ Frontier Research System, The Institute of Physical and Chemical Research (RIKEN), Wako-shi, Saitama 351-0198, Japan \\ ${ }^{3}$ NEC Fundamental Research Laboratories, Tsukuba, Ibaraki 305-8051, Japan \\ ${ }^{4}$ Center for Theoretical Physics, Physics Department, Center for the Study of Complex Systems, \\ The University of Michigan, Ann Arbor, Michigan 48109-1040, USA
}

(Dated: November 23, 2018)

\begin{abstract}
Exploiting the intrinsic nonlinearity of superconducting Josephson junctions, we propose a scalable circuit with superconducting qubits (SCQs) which is very similar to the successful one now being used for trapped ions. The SCQs are coupled to the "vibrational" mode provided by a superconducting LC circuit or its equivalent (e.g., a SQUID). Both single-qubit rotations and qubit-LC-circuit couplings/decouplings can be controlled by the frequencies of the time-dependent magnetic fluxes. The circuit is scalable since the qubit-qubit interactions, mediated by the LC circuit, can be selectively performed, and the information transfer can be realized in a controllable way.

PACS numbers: 03.67.Lx, 74.50.+r, 85.25.Cp
\end{abstract}

\section{INTRODUCTION}

Superconducting quantum circuits with Josephson junctions are currently studied for their potential applications in quantum information processing [1]. Quantum coherent oscillations and conditional gate operations have been demonstrated using two coupled superconducting charge qubits [2, 3]. For a circuit with two coupled flux qubits, spectroscopic measurements show that it acts as a quantized four-level system [4]. Further, entanglement has been experimentally verified in coupled flux [5] and phase [6, 7, 8] qubits.

A major challenge for superconducting qubits (SCQs) is how to design an experimentally realizable circuit where the couplings for different qubits can be selectively switched on and off, and then scaled up to many qubits. Although twoqubit gates can be generated (see, e.g., Ref. [9]) with alwayson interbit couplings, it is still very difficult to scale up experimental circuits $[2,3,4,5,6,6,8]$. Theoretically, the circuits (e.g., Refs. [10, 11, 12, 13, 14, 15, 16, 17, 18]) can be scaled up via a common data bus (DB). The DB modes are virtually excited (e.g., Refs. [10, 11]]) or excited (e.g., Refs. [12, 13, 14, 15, 16, 17, 18]). In the former case [10, 11], the effective qubit couplings can be switched on and off by changing the magnetic flux through the circuit within nanoseconds, which is a challenge for current experiments. In the later case [12, 13, 14, 15, 16, 17], the qubit and the DB are coupled or decoupled when they have the same (resonant) or different (non-resonant) frequencies, realized via a sudden non-adiabatic change of either the qubit or the DB eigenfrequency. This introduces additional noise.

The superconducting Josephson junction is a key building block of superconducting quantum circuits. Nonlinearity is its intrinsic characteristic. This nonlinearity can be used to adjust the inter-qubit couplings [19, 20, 21, 22] by changing the current bias of the coupler, and thus cancelling the direct mutual inductance between the qubits. It can also be used to realize the switchable coupling between two inductively coupled superconducting flux qubits via a variable-frequency magnetic flux [23]. Also, recently, the level quantization of the LC cir- cuit has been experimentally demonstrated [24, 25].

Combining the variable-frequency-controlled coupling approach [23] and experimental achievements of the quantum LC circuit [24, 25], we now study a different approach to realize scalable SCQs via a common DB, which is either a quantum LC circuit or its equivalent, modeled by a harmonic oscillator [24, 25]. The equivalent LC circuits can be either a cavity field (e.g., [12, 13, 14]) realized by, for instance, a one-dimensional transmission line resonator [16] or a superconducting loop with Josephson junctions (e.g., a dc-based SQUID). More significantly, all SCQs can work at their optimal points when the data bus is a superconducting loop with Josephson junctions (this is not the case with standard LC DBs). In our this novel approach, the individual properties (e.g., eigenfrequencies) of the DB and SCQs are always fixed, but the SCQ-DB couplings can be conveniently controlled by changing the frequencies of the applied time-dependent magnetic fluxes (TDMFs). This is promising, because it is often easier to produce fast and precise frequency shifts of the radio-frequency control signal in experiments, as opposed to changing the amplitude of the dc signal.

We should point out that in our proposal the quantum LC circuit or its equivalent has to be excited when the information is transferred from one qubit to another; therefore it is an active element, not a passive one which is just virtually excited. Our proposal can be essentially reduced to the one used for trapped ions [26]. The SCQs are coupled to the "vibrational" mode provided by a superconducting LC circuit or its equivalent (e.g., a SQUID). Both single-qubit rotations and qubitLC-circuit couplings/decouplings can be controlled by the frequencies of the time-dependent magnetic fluxes. It means that SQCs can be coupled and separately addressed similarly to trapped ions. This similarity is significant because trapped ions [26] are further ahead, along the quantum computing roadmap. It is important to stress that our theoretical model can well explain the blue/red sideband excitations which have been experimentally implemented in superconducting qubit circuits [24, 27]. 

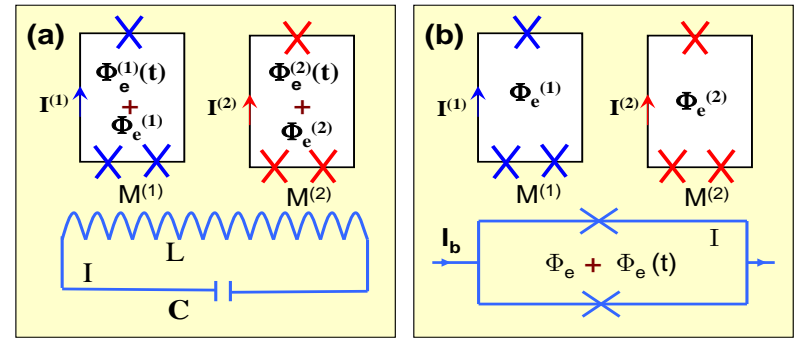

FIG. 1: (Color online) The $l$ th flux qubit with three junctions is coupled to an LC circuit in (a) or a dc-biased SQUID with biased current $I_{b}$ in (b) by the mutual inductance $M^{(l)}(l=1,2)$. (a) An externally applied magnetic flux through the $l$ th qubit loop includes a dc $\Phi_{e}^{(l)}$ term and ac $\Phi_{e}^{(l)}(t)$ term controlling the coupling in (a). The currents through the first qubit, second qubit, and LC circuit in (a) [or SQUID loop in (b)] are $I^{(1)}, I^{(2)}$, and $I$ respectively. (b) However, when a dc-biased SQUID forms an equivalent LC circuit, the SQUID-qubit couplings are controlled by a TDMF, $\Phi_{e}(t)=A \cos \left(\omega_{c} t\right)$, through the SQUID loop. The TDMF is added to the nonlinear qubit in (a) and to the nonlinear SQUID loop in (b). The configuration in (b) is significantly better, because both qubits can work at the optimal point $f=1 / 2$.

\section{MODEL}

We study three-junction flux qubits (e.g., [28, 29]). As shown in Fig. 1, we consider the simplest circuit, where two flux qubits are coupled to a DB: either an LC circuit or a superconducting loop with junctions (e.g., a dc-biased SQUID). Without loss of generality and for simplicity, the DB is here assumed to be an LC circuit with an inductance $L$ and a capacitance $C$. The mutual inductance between the $l$ th qubit and the LC circuit is $M^{(l)}(l=1,2)$. The applied magnetic flux $\Phi^{(l)}$ through the $l$ th qubit loop in Fig. 1(a) is assumed to include a static (or dc) magnetic flux $\Phi_{\mathrm{e}}^{(l)}$, and also a TDMF

$$
\Phi_{\mathrm{e}}^{(l)}(t)=A_{l} \cos \left(\omega_{c}^{(l)} t\right),
$$

which controls the qubit-DB couplings. Neglecting the mutual inductance between the two qubits, the Hamiltonian can be written as

$$
H=\sum_{l=1}^{2} H_{l}+\frac{Q^{2}}{2 C}+\frac{\phi^{2}}{2 L}+\sum_{l=1}^{2} I M^{(l)} I^{(l)},
$$

with the current $I$ and magnetic flux $\phi=I L$ through the LC circuit loop. Considering a three-junction qubit, the singlequbit Hamiltonian $H_{l}$ in Eq. (2) should be

$$
H_{l}=\sum_{i=1}^{3} \frac{\Phi_{0}}{2 \pi}\left[\frac{\Phi_{0} C_{\mathrm{J} i}^{(l)}}{\pi}\left(\dot{\varphi}_{i}^{(l)}\right)^{2}-I_{0 i}^{(l)} \cos \varphi_{i}^{(l)}\right],
$$

after neglecting the qubit self-inductance and constant terms $I_{0 i}^{(l)} \Phi_{0} / 2 \pi \equiv E_{\mathrm{J} i}^{(l)}$. Each junction in the $l$ th qubit has a capacitance $C_{\mathrm{J} i}^{(l)}$, phase drop $\varphi_{i}^{(l)}$, and supercurrent $I_{i}^{(l)}=$
$I_{0 i}^{(l)} \sin \varphi_{i}^{(l)}$, with critical current $I_{0 i}^{(l)}$. The loop current of the $l$ th qubit is

$$
I^{(l)}=C_{l} \sum_{i=1}^{3} \frac{I_{0 i}^{(l)}}{C_{\mathrm{J} i}^{(l)}} \sin \varphi_{i}^{(l)},
$$

where

$$
\frac{1}{C_{l}}=\frac{1}{C_{\mathrm{J} 1}^{(l)}}+\frac{1}{C_{\mathrm{J} 2}^{(l)}}+\frac{1}{C_{\mathrm{J} 3}^{(l)}},
$$

with the convention $C_{\mathrm{J} 3}^{(l)}=\alpha_{l} C_{\mathrm{J} 1}^{(l)}=\alpha_{l} C_{\mathrm{J} 2}^{(l)}$, and $\alpha_{l}<1$. The LC circuit can be modeled by a harmonic oscillator described by the creation operator

$$
a^{\dagger}=\frac{1}{\sqrt{2 \hbar \omega C}}(\omega C \phi-i Q)
$$

and its conjugate $a$, with frequency $\omega=1 / \sqrt{L C}$. Considering the TDMF, the phase constraint condition [28] through the lth qubit loop becomes

$$
\sum_{i=1}^{3} \varphi_{i}^{(l)}+2 \pi\left[f+\frac{\Phi_{e}^{(l)}(t)}{\Phi_{0}}\right]=0
$$

with the reduced bias flux

$$
f=\left(\Phi_{e}^{(l)}-M^{(l)} I\right) / \Phi_{0} .
$$

Here, the bias $f$ includes the flux $M^{(l)} I$, produced by the LC circuit. Thus, in the qubit basis, Eq. (2) becomes

$$
\begin{aligned}
H & =\frac{\hbar}{2} \sum_{l=1}^{2} \omega_{q}^{(l)} \sigma_{z}^{(l)}+\hbar \omega a^{\dagger} a+\sum_{l=1}^{2} H_{\mathrm{int}}^{(l)} \\
& +\sum_{l=1}^{2}\left(\lambda_{l} \sigma_{-}^{(l)}+\text { h.c. }\right) \cos \left(\omega_{c}^{(l)} t\right) \\
& -\sum_{l=1}^{2}\left(a^{\dagger}+a\right)\left(\Omega_{l} \sigma_{-}^{(l)}+\text { h.c. }\right) \cos \left(\omega_{c}^{(l)} t\right)
\end{aligned}
$$

after neglecting the constant terms. Here the Pauli operators of the $l$ th qubit are defined by $\sigma_{+}^{(l)}=\left|e_{l}\right\rangle\left\langle g_{l}\left|, \sigma_{-}^{(l)}=\right| g_{l}\right\rangle\left\langle e_{l}\right|$, and $\sigma_{z}^{(l)}=\left|e_{l}\right\rangle\left\langle e_{l}|-| g_{l}\right\rangle\left\langle g_{l}\right|$. The computational basis states of the $l$ th qubit are defined [28, 29], for $\Phi_{e}^{(l)}(t)=0$, by the two lowest eigenstates, $|0\rangle_{l}=\left|g_{l}\right\rangle$, and $|1\rangle_{l}=\left|e_{l}\right\rangle$, of $H_{l}$ with the two independent variables $\varphi_{p}^{(l)}=\left(\varphi_{1}^{(l)}+\varphi_{2}^{(l)}\right) / 2$ and $\varphi_{m}^{(l)}=\left(\varphi_{1}^{(l)}-\varphi_{2}^{(l)}\right) / 2$.

The first two terms in Eq. (9) denote the free Hamiltonians of both qubits and the LC circuit; $\omega_{q}^{(l)}$ is the transition frequency of the $l$ th qubit. The always-on interaction Hamiltonian between the $l$ th qubit and the DB in the third term of Eq. (9) is

$$
H_{\mathrm{int}}^{(l)}=\left(a^{\dagger}+a\right)\left(G_{l} \sigma_{-}^{(l)}+\text { h.c }\right)
$$

with the coupling constant

$$
G_{l}=M^{(l)} \sqrt{\frac{\hbar \omega}{2 L}}\left\langle e_{l}\left|I_{0}^{(l)}\right| g_{l}\right\rangle .
$$


The fourth term in Eq. (9) represents the interaction between the $l$ th qubit and its TDMF with the interaction strength

$$
\lambda_{l}=A_{l}\left\langle e_{l}\left|I_{3}^{(l)}\right| g_{l}\right\rangle
$$

The fifth term of Eq. (9) is the controllable nonlinear interaction among the $l$ th qubit, the DB, and the TDMF, with the coupling strength

$$
\Omega_{l}=\frac{4 \pi^{2} A_{l} M^{(l)} C_{l}}{C_{\mathrm{J} 3}^{(l)} \Phi_{0}^{2}} \sqrt{\frac{\hbar \omega}{2 L}}\left\langle e_{l}\left|E_{\mathrm{J} 3}^{(l)} \cos \varphi_{3}^{(l)}\right| g_{l}\right\rangle .
$$

This nonlinear interaction term between the $l$ th qubit, the DB, and the TDMF originates from the expansion of the loop current $I^{(l)}$ of the $l$ th qubit in Eq. (4) to first order on the small reduced flux $\Phi_{e}^{(l)}(t) / \Phi_{0}$ via the phase constrain condition in Eq. (7). Above, the TDMF $\Phi_{e}^{(l)}(t)$ equals zero when calculating the coupling strengths $G_{l}, \lambda_{l}$, and $\Omega_{l}$. That is, $I_{0}^{(l)}$ and $I_{3}^{(l)}$ are supercurrents through the loop and the third junction respectively when $\Phi_{e}^{(l)}(t)=0$.

\section{SWITCHABLE INTERACTION BETWEEN QUBIT AND DATA BUS}

We find that the Hamiltonian (9) can be reduced to the one used in trapped ions [26] if the always-on interaction terms $H_{\text {int }}^{(l)}$ can be neglected. This approximation is valid [8] during the TDMF operations, in the large detuning regime between any qubit (e.g., lth qubit) and the DB

$$
\Delta_{l}=\omega_{q}^{(l)}-\omega \gg\left|G_{l}\right|
$$

which can be achieved when the circuit is initially fabricated. Thus, neglecting the always-on coupling $H_{\text {int }}^{(l)}$ between the data bus and the qubits, the Hamiltonian (9) is reduced to

$$
\begin{aligned}
H & =\hbar \omega a^{\dagger} a+\frac{\hbar}{2} \sum_{l=1}^{2} \omega_{q}^{(l)} \sigma_{z}^{(l)} \\
& +\sum_{l=1}^{2}\left(\lambda_{l} \sigma_{-}^{(l)}+\text { h.c. }\right) \cos \left(\omega_{c}^{(l)} t\right) \\
& -\sum_{l=1}^{2}\left(a^{\dagger}+a\right)\left(\Omega_{l} \sigma_{-}^{(l)}+\text { h.c. }\right) \cos \left(\omega_{c}^{(l)} t\right)
\end{aligned}
$$

which now has the same form as the one used for quantum computing with trapped ions, in the standard Lamb-Dicke limit (see, e.g., [26]).

Therefore, the essential difference between our Hamiltonian in Eq. (9) and the one used for experiments [16, 24, 25] is that: (a) the nonlinear coupling between the data bus, qubits, and the classical field in Eq. (9) is very important for the superconducting case. Using this term, we can explain the sideband transitions in the experiments [24, 25]; (b) the always-on coupling $H_{\text {int }}^{(l)}$ between the qubits and the data bus should be negligibly small in our proposal. Our theoretical model is in contrast with those in Refs. [24, 25] where: (a) there is no nonlinear coupling between the data bus, qubits, and the classical field; (b) the always-on Hamiltonian $H_{\text {int }}^{(l)}$ could not be neglected. That is, in Refs. [24, 25], the Hamiltonian is just the usual Jaynes-Cummings model which cannot be directly used to explain the sideband excitations, especially for the experimental results in Refs. [24, 27].

Analogous to the case of trapped ions, in our proposed devices, three-types of dynamical evolutions (carrier process; red sideband excitation; and blue sideband excitation) can be produced by the TDMF using the frequency-matching (resonant) condition and neglecting all fast oscillating terms. These three dynamical evolutions can be described as follows.

(i) If $\omega_{c}^{(l)}=\omega_{q}^{(l)}$, the qubit and the DB evolve independently in the large detuning condition. The external flux $\Phi_{e}^{(l)}(t)$ is only used to separately address the $l$ th qubit rotations. These rotations are governed by the Hamiltonian

$$
H_{c}^{(l)}=\lambda_{l} \sigma_{-}^{(l)}+\text { h.c., }
$$

in the interaction picture and using the rotating-wave approximation (RWA) (also for the $H_{r}^{(l)}$ and $H_{b}^{(l)}$ shown below). This is the so-called carrier process in the trapped ions approach.

(ii) If the frequencies satisfy the condition $\omega_{c}^{(l)}=\omega_{q}^{(l)}-\omega$, then the $\Phi_{e}^{(l)}(t)$ assists the $l$ th qubit to couple resonantly with the DB. This is the red sideband excitation, governed by the Hamiltonian

$$
H_{r}^{(l)}=\Omega_{l} a^{\dagger} \sigma_{-}^{(l)}+\text { h.c.. }
$$

(iii) In the blue sideband excitation, the frequencies satisfy the condition $\omega_{c}^{(l)}=\omega_{q}^{(l)}+\omega$, with the Hamiltonian

$$
H_{b}^{(l)}=\Omega_{l} a \sigma_{-}^{(l)}+\text { h.c.. }
$$

Based on above discussions, it can be easily found that our derived Hamiltonian in Eq. (9), reduced to Eq. (15), can naturally explain experimental results on the sideband excitations. For example, in Ref. [27], the qubit and the DB frequencies are $14 \mathrm{GHz}$ and $4.3 \mathrm{GHz}$, respectively; the frequency $\omega_{c}$ for the red or blue sideband excitation is $9.7 \mathrm{GHz}$ or $18.32 \mathrm{GHz}$. However, the Jaynes-Cummings model cannot be used to explain these experiments. The qubit-DB couplings/decouplings can be controlled by appropriately selecting the $\omega_{c}^{(l)}$ of $\Phi_{e}^{(l)}(t)$ to match/mismatch the above frequency conditions of the sideband excitations.

\section{SINGLE- AND TWO-QUBIT GATES}

For the $l$ th qubit, the carrier process described by $H_{c}^{(l)}$ can be used to perform the single-qubit rotation

$$
U_{c}^{(l)}\left(\beta_{l}, \phi_{l}\right)=\exp \left[-i \frac{\beta_{l}}{2}\left(e^{-i \phi_{l}} \sigma_{-}^{(l)}+e^{i \phi_{l}} \sigma_{+}^{(l)}\right)\right] .
$$

Here, $\beta_{l}=\left|\lambda_{l}\right| \tau / \hbar$ depends on the Rabi frequency $\left|\lambda_{l}\right| / \hbar$ and duration $\tau ; \phi_{l}$ is related to the phase of the TDMF applied to 
the $l$ th qubit. For example, the phases $\phi_{l}=0$ and $\phi_{l}=3 \pi / 2$ correspond to the rotations $R_{x}^{(l)}\left(\beta_{l}\right)$ and $R_{y}^{(l)}\left(\beta_{l}\right)$, about the $x$ and $y$ axis, respectively. Thus, any single-qubit operation can be realized by a series of $R_{x}^{(l)}\left(\beta_{l}\right)$ and $R_{y}^{(l)}\left(\beta_{l}\right)$ rotations with well-chosen different angles $\beta_{l}$.

Two-qubit gates can be obtained using two qubits interacting sequentially with their DB as in Ref. [26]. There, the controlled phase-flip and the controlled-NOT gates can be obtained by three and five steps, respectively. Here, we only discuss the difference between our proposal and the one used for trapped ions. In our proposed circuit, the ratio $\left|G_{l}\right| / \Delta_{l}$ cannot be infinitely small. Then, the uncontrollable qubitDB interaction $H_{\text {int }}^{(l)}$ needs to be considered by the effective Hamiltonian [30]

$$
H_{e}^{(l)}=\hbar \frac{\left|G_{l}\right|^{2}}{\Delta_{l}}\left[\left|e_{l}\right\rangle\left\langle e_{l}\left|a a^{\dagger}-\right| g_{l}\right\rangle\left\langle g_{l}\right| a^{\dagger} a\right]
$$

when the $l$ th qubit is not addressed by the TDMF. After including this effect, three pulses (successively applied to the first, second, and first qubits) with durations $\tau_{1}, \tau_{2}$, and $\tau_{3}$ (used to perform a controlled phase-flip gate in Ref. [26]) will result in a two-qubit gate $U_{\text {two }}$. This can be expressed as

$$
U_{\mathrm{two}}=\left(\begin{array}{cccc}
1 & 0 & 0 & 0 \\
0 & \exp \left(-i \theta_{1}\right) & 0 & 0 \\
0 & 0 & \exp \left(i \theta_{2}\right) & 0 \\
0 & 0 & 0 & -\exp \left(-i \theta_{3}\right)
\end{array}\right)
$$

in the two-qubit basis $\left\{\left|g_{1}\right\rangle\left|g_{2}\right\rangle,\left|g_{1}\right\rangle\left|e_{2}\right\rangle,\left|e_{1}\right\rangle\left|g_{2}\right\rangle,\left|e_{1}\right\rangle\left|e_{2}\right\rangle\right\}$ with the parameters

$$
\begin{aligned}
\theta_{1} & =\frac{2\left|G_{2}\right|^{2}}{\Delta_{2}} \tau_{1}, \\
\theta_{2} & =\frac{\left|G_{2}\right|^{2}}{\Delta_{2}} \tau_{1}+\frac{\left|G_{1}\right|^{2}}{\Delta_{1}} \tau_{2}, \\
\theta_{3} & =\frac{3\left|G_{2}\right|^{2}}{\Delta_{2}} \tau_{1}+\frac{\left|G_{1}\right|^{2}}{\Delta_{1}} \tau_{2} .
\end{aligned}
$$

The two-qubit gate $U_{\text {two }}$ in Eq. (21) is just a controlled phaseflip gate when the large detuning condition $\left|G_{l}\right| / \Delta_{l} \sim 0$ is satisfied. Moreover, any quantum operation can also be realized by combining the two-qubit gate $U_{\text {two }}$ with other singlequbit operations.

\section{ENTANGLEMENT AND STATE TRANSFER}

We now consider two different external fields satisfying frequency-matching conditions, e.g., for the red sideband excitation, which are simultaneously applied to the two qubits in Fig. 1. Then, in the interaction picture and the RWA, the interaction Hamiltonian in Eq. (9), between the LC circuit and the two qubits, becomes

$$
H_{1}=\sum_{l=1}^{2}\left(\Omega a^{\dagger} \sigma_{-}+\text {h.c. }\right)
$$

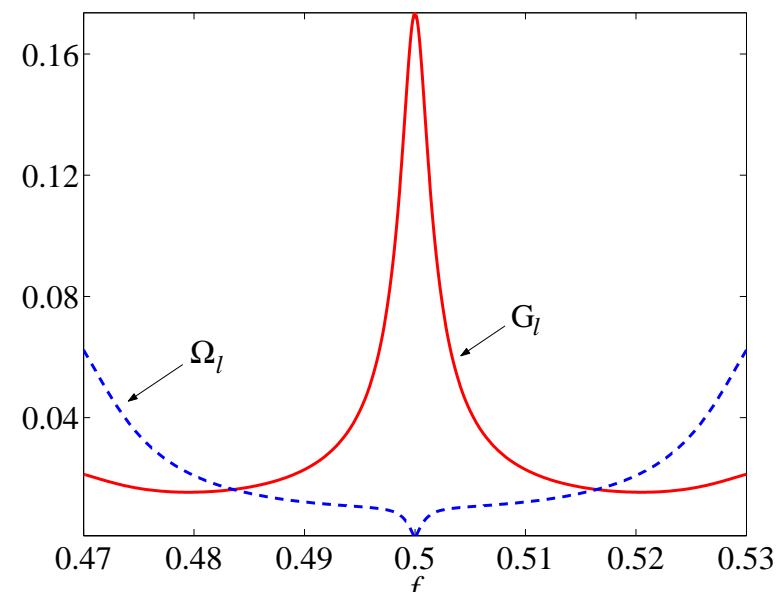

FIG. 2: (Color online) Plots of the $f$-dependent always-on qubitDB coupling strength $G_{l}$ (red curve) and the TDMF-controlled qubit-DB coupling strength $\Omega_{l}$ (blue curve), rescaled by $R_{l}=$ $\left(2 \pi / \Phi_{0}\right) M^{(l)} E_{\mathrm{J} 3}^{(l)} \sqrt{\hbar \omega / 2 L}$.

For simplicity, the coupling strengths between the LC circuit and different qubits are now assumed to be identical, e.g., $\Omega_{1}=\Omega_{2}=|\Omega| e^{-i \theta}$. If the LC circuit is initially prepared in the first excited state $|1\rangle$, then the wave-function $|\Psi(t)\rangle$ of the whole system can be written as

$$
\begin{aligned}
|\Psi(t)\rangle & =-i e^{i \theta} \sin (\sqrt{2} \Omega t)\left[\left|e_{1}\right\rangle\left|g_{2}\right\rangle|0\rangle+\left|g_{1}\right\rangle\left|e_{2}\right\rangle|0\rangle\right] \\
& +\cos (\sqrt{2} \Omega t)\left|g_{1}\right\rangle\left|g_{2}\right\rangle|1\rangle
\end{aligned}
$$

When $\sqrt{2} \Omega t / \hbar=\pi / 2$, then the LC circuit is in the vacuum state $|0\rangle$ and a maximally entangled state between two qubits can be generated as

$$
\left|\Psi^{+}\right\rangle_{12}=\frac{1}{\sqrt{2}}\left[\left|e_{1}\right\rangle\left|g_{2}\right\rangle+\left|g_{1}\right\rangle\left|e_{2}\right\rangle\right]
$$

When adding one more qubit to Fig. 1(a) or (b), an unknown state $|\psi\rangle=\beta_{1}\left|g_{1}\right\rangle+\beta_{2}\left|e_{1}\right\rangle$ in the first qubit can be transferred to the third one using the standard teleportation procedure: i) a maximally entangled state $\left|\Psi^{+}\right\rangle_{23}=$ $\left[\left|e_{2}\right\rangle\left|g_{3}\right\rangle+\left|g_{2}\right\rangle\left|e_{3}\right\rangle\right] / \sqrt{2}$ between the second and third qubits is prepared using the same method outlined above; ii) a CNOT gate $U_{\mathrm{CNOT}}^{(12)}$ is implemented for the first and second qubits (here, the second one is the target); iii) a Hadmard gate is implemented on the first one; iv) a simultaneous measurement, which can now be done experimentally [8], is performed on the first and the second qubits. The four different measured results $\left\{\left|e_{1}, e_{2}\right\rangle,\left|e_{1}, g_{2}\right\rangle,\left|g_{1}, e_{2}\right\rangle\right.$, and $\left.\left|g_{1}, g_{2}\right\rangle\right\}$ correspond to four outputs $\left\{\left|\psi_{1}\right\rangle,\left|\psi_{2}\right\rangle,\left|\psi_{3}\right\rangle\right.$, and $\left.\left|\psi_{3}\right\rangle\right\}$ in the third qubit. The unknown state in the first qubit can be transferred to the third one when the measured result for the first and second qubits is $\left|e_{1}, e_{2}\right\rangle$. However, appropriate gates (i.e., $\sigma_{x}^{(3)}, \sigma_{z}^{(3)}$, and $\sigma_{z}^{(3)} \sigma_{x}^{(3)}$ ) need to be performed on the other three outputs mentioned above to transfer $|\psi\rangle$ to the third qubit. 


\section{EXPERIMENTALLY ACCESSIBLE PARAMETERS}

We now analyze the coupling constants related to the $l$ th qubit: (i) the always-on qubit-DB coupling strength

$$
G_{l} \propto\left\langle e_{l}\left|I_{0}^{(l)}\right| g_{l}\right\rangle
$$

and (ii) the TDMF-controlled qubit-DB coupling strength

$$
\Omega_{l} \propto\left\langle e_{l}\left|\cos \left(2 \varphi_{p}+2 \pi f\right)\right| g_{l}\right\rangle .
$$

At the degeneracy point $f=1 / 2$, the qubit potential is symmetric [29] and its ground and excited states have opposite parities; however, $\cos \left(2 \varphi_{p}+2 \pi f\right)$ and the qubit loop current $I_{0}^{(l)}$ have even and odd parities, respectively. Therefore $\Omega_{l}=0$ but $G_{l} \neq 0$ when $f=1 / 2$. Clearly, that $\Omega_{l}=0$ can be avoided by slightly shifting $f$ away from the degeneracy point. The experiments on sideband excitations, e.g., in Refs. [24, 27], were performed with $f \neq 1 / 2$. Moreover, the controlled phase-flip gate [26], requiring a transition from the ground state to the second excited state, also implies that the reduced bias [29] flux $f \neq 1 / 2$.

Figure 2 shows the $f$-dependent coupling strengths $G_{l}$ and $\Omega_{l}$, rescaled by

$$
R_{l}=\left(2 \pi / \Phi_{0}\right) M^{(l)} E_{\mathrm{J} 3}^{(l)} \sqrt{\hbar \omega / 2 L} .
$$

As in Ref. [24], the Josephson energies of the two bigger junctions of the $l$ th qubit are $E_{\mathrm{J} 1}^{(l)}=E_{\mathrm{J} 2}^{(l)}=225 \mathrm{GHz}$ and the ratio between the small and big junction areas is $\alpha_{l}=0.76$. The ratio between the Josephson energy $E_{J 1}^{(l)}$ and the charge energy $E_{c}^{(l)}$ of the $l$ th qubit is about 30.8 . Using the qubit parameters listed above and also taking the amplitude $A_{l}=\Phi_{0} / 30$ of the TDMF applied to the $l$ th qubit, Fig. 2] shows that $G_{l}$ and $\Omega_{l}$ are comparable when $f$ is away from $1 / 2$; e.g., $G_{l} \approx 0.0579 R_{l}$ and $\Omega_{l} \approx 0.0224 R_{l}$ when $f=0.49$. The strength $\Omega_{l}$ can also be larger than the strength $G_{l}$ in the range, e.g., $0.47 \lesssim f \lesssim 0.477$.

If the capacitance and inductance of the LC circuit is taken [24] as $12 \mathrm{pF}$ and $250 \mathrm{pH}$, then the frequency $\omega$ of the LC circuit is about $2.9 \mathrm{GHz}$. When the mutual inductance $M^{(l)}$ between the $l$ th qubit and the LC circuit is taken as $20 \mathrm{pH}$, then $G_{l} \approx 37.6 \mathrm{MHz}$ and $\Omega_{l} \approx 14.6 \mathrm{MHz}$ when $f=0.49$. The $l$ th qubit frequency computed is about 18 $\mathrm{GHz}$ when $f=0.49$. Therefore, the detuning between the $l$ th qubit and the LC circuit is $\Delta_{l} \approx 15.1 \mathrm{GHz}$, and the ratio $G_{l} / \Delta_{l} \approx 0.0015$. Indeed, the always-on coupling $G_{l}$ is negligibly small when the $l$ th qubit works at $f=0.49$ for measuring the sideband excitations. The phase corrections $\theta_{i}$ in Eq. 21) should be very small with short operation times for those qubits when no $\Phi_{e}^{(l)}(t)$ is applied.

For the LC circuit, if its capacitance $C$ and inductance $L$ are assumed as $\sim 1 \mathrm{pF}$ and $\sim 10 \mathrm{nH}$, respectively, then the LC circuit plasma frequency can be $\sim 1.6 \mathrm{GHz}$. The linear dimension for the LC circuit can be $\lesssim 1 \mathrm{~cm}$. The estimated distance for a negligible mutual inductance between two nearest qubits is $\sim 200 \mu \mathrm{m}$, and thus one DB can approximately interact with $\sim 40$ qubits. Of course, the larger $L$ of the LC circuit could have a larger linear dimension (allowing, e.g., $L \sim 100 \mathrm{nH}$ ), and then more qubits, here about 400, could interact with the LC circuit. In practice, the superpositions of the ground and excited states for an LC circuit decay on a time scale given by $1 / R C$, here $R$ is the residual resistance of the circuit and its radiation losses.

\section{DISCUSSIONS AND CONCLUSIONS}

For flux qubits, the single-qubit states can be measured by using, e.g., either a tank circuit weakly coupled to the qubit [5] or a dc-SQUID [31]. If only a single-qubit measurement can be done at a time [32] or simultaneous measurements can be done (e.g., as for phase qubits [33]), then any unknown quantum state can be reconstructed [32, 33] and the information of the qubits can be read out.

In our proposal, two crucial points are: (1) the qubit and the LC circuit data bus should initially have a large detuning, such that their always-on coupling is negligibly small when the TDMF-assisted qubit-DB coupling is implemented; (2) the nonlinearity of the JJs is essential to achieve our goal: the nonlinear coupling between these three: the qubit, DB, and TDMF. Based on these two requirements, the circuit can be modified according to different experimental setups, e.g., the LC circuit can be replaced by a superconducting loop with JJs (e.g., a dc-biased SQUID as in Fig. 1(b)). Threejunction flux qubits can also be replaced by other qubits [1], e.g., one- or four-junction flux qubits, phase qubits, or chargeflux qubits. Although the self-inductances of the qubits are neglected here, our method is still valid for the qubits with nonzero self-inductances [34].

Our numerical calculations show that the TDMF-controlled coupling strength $\Omega_{l}$ is not large enough to realize very fast two-qubit operations when the DB is a simple LC circuit. In principle, this problem could be solved by using a superconducting loop with Josephson junctions (e.g., dc-SQUID in Ref. [19]) as a data bus instead of a simple LC circuit. Thus, the TDMF can be applied to the DB loop and the qubit can work at the optimal point; the DB-qubit always-on coupling can be minimized to zero; the TDMF-controlled couplingstrength can be large enough to realize fast two-qubit operations. A more detailed study on this issue will be presented elsewhere.

In conclusions, using the nonlinearity of the superconducting JJs, we theoretically explain the sideband excitations for qubits coupled to an LC circuit and show how to scale these to many qubits. In contrast to previous proposals (e.g., [10, 11, 16, 17]), the properties (e.g., eigenfrequencies) of the qubits and the DB are fixed when processing either the resonant coupling or the non-resonant decoupling. Also, the qubit-DB couplings/decouplings are controlled neither by changing the magnetic flux through the loop nor by changing the eigenfrequencies of the qubits (or the DB). They are only controlled via the frequency shifts of TDMFs, which is much easier to achieve experimentally. 


\section{Acknowledgments}

We thank J.Q. You, Y. Nakamura, Y.A. Pashkin, O. Astafiev, and K. Harrabi for discussions. FN was supported in part by the US National Security Agency (NSA), Army Research Office (ARO), Laboratory of Physical Sciences (LPS), and the National Science Foundation grant No. EIA-0130383.
[1] J.Q. You and F. Nori, Phys. Today 58 (11), 42 (2005).

[2] Yu. A. Pashkin, T. Yamamoto, O. Astafiev, Y. Nakamura, D.V. Averin, and J.S. Tsai, Nature 421, 823 (2003).

[3] T. Yamamoto, Yu. A. Pashkin, O. Astafiev, Y. Nakamura, and J.S. Tsai, Nature 425, 941 (2003).

[4] J.B. Majer, F.G. Paauw, A.C.J. ter Haar, C.J.P.M. Harmans, and J.E. Mooij, Phys. Rev. Lett. 94, 090501 (2005).

[5] A. Izmalkov, M. Grajcar, E. Il'ichev, Th. Wagner, H.-G. Meyer, A. Yu. Smirnov, M.H.S. Amin, Alec Maassen van den Brink, and A.M. Zagoskin, Phys. Rev. Lett. 93, 037003 (2004).

[6] H. Xu, F.W. Strauch, S.K. Dutta,P. R. Johnson, R.C. Ramos, A. J. Berkley, H. Paik, J.R. Anderson, A.J. Dragt, C.J. Lobb, and F.C. Wellstood, Phys. Rev. Lett. 94, 027003 (2005).

[7] A.J. Berkley, H. Xu, R.C. Ramos, M.A. Gubrud, F.W. Strauch, P.R. Johnson, J.R. Anderson, A.J. Dragt, C.J. Lobb, and F.C. Wellstood, Science 300, 1548 (2003).

[8] R. McDermott, R.W. Simmonds, M. Steffen, K.B. Cooper, K. Cicak, K.D. Osborn, S. Oh, D.P. Pappas, and J.M. Martinis, Science 307, 1299 (2005).

[9] F.W. Strauch, P.R. Johnson, A.J. Dragt, C.J. Lobb, J.R. Anderson, and F.C. Wellstood, Phys. Rev. Lett. 91, 167005 (2003).

[10] Y. Makhlin, G. Schön, and A. Shnirman, Rev. Mod. Phys. 73, 357 (2001).

[11] J.Q. You, J.S. Tsai, and F. Nori, Phys. Rev. Lett. 89, 197902 (2002).

[12] J.Q. You and F. Nori, Phys. Rev. B 68, 064509 (2003).

[13] J.Q. You, J.S. Tsai, and F. Nori, Phys. Rev. B 68, 024510 (2003).

[14] Y.X. Liu, L.F. Wei, and F. Nori, Europhy. Lett. 67, 941 (2004); Phys. Rev. A 71, 063820 (2005).

[15] A. Blais, R.-S. Huang, A. Wallraff, S.M. Girvin, and R.J. Schoelkopf, Phys. Rev. A 69, 062320 (2004).

[16] A. Wallraff, D.I. Schuster, A. Blais, L. Frunzio, R.-S. Huang, J. Majer, S. Kumar, S.M. Girvin, and R.J. Schoelkopf, Nature 431, 162 (2004).

[17] A.N. Cleland and M.R. Geller, Phys. Rev. Lett. 93, 070501 (2004).
[18] L.F. Wei, Y.X. Liu, and F. Nori, Phys. Rev. B 71, 134506 (2005).

[19] B.L.T. Plourde, J. Zhang, K.B. Whaley, F.K. Wilhelm, T.L. Robertson, T. Hime, S. Linzen, P.A. Reichardt, C.-E. Wu, and J. Clarke, Phys. Rev. B 70, 140501(R) (2005).

[20] B.L.T. Plourde, T.L. Robertson, P.A. Reichardt, T. Hime, S. Linzen, C.-E. Wu, and J. Clarke, Phys. Rev. B 72, 060506 (2005).

[21] T.L. Robertson, B.L.T. Plourde, P.A. Reichardt, T. Hime, C.-E. Wu, and J. Clarke, Phys. Rev. B 73, 174526 (2006).

[22] M.D. Kim, Phys. Rev. B 74, 184501 (2006).

[23] Y.X. Liu, L.F. Wei, J.S. Tsai, and F. Nori, Phys. Rev. Lett. 96, 067003 (2006).

[24] I. Chiorescu, P. Bertet, K. Semba, Y. Nakamura, C.J.P.M. Harmans, and J.E. Mooij, Nature 431, 159 (2004).

[25] J. Johansson, S. Saito, T. Meno, H. Nakano, M. Ueda, K. Semba, and H. Takayanagi, Phys. Rev. Lett. 96, 127006 (2006).

[26] M. Sasura and V. Buzek, J. Mod. Opt. 49, 1593 (2002).

[27] K. Semba, S. Saito, T. Meno, J. Johansson, and H. Takayanagi, Proc. of 24th Inter. Conf. on Low Temp. Phy., Florida, 939 (2005).

[28] T.P. Orlando, J.E. Mooij, L. Tian, C.H. van der Wal, L.S. Levitov, S. Lloyd, and J.J. Mazo, Phys. Rev. B 60, 15398 (1999).

[29] Y.X. Liu, J.Q. You, L.F. Wei, C.P. Sun, and F. Nori, Phys. Rev. Lett. 95, 087001 (2005).

[30] Y.X. Liu, L.F. Wei, and F. Nori, Phys. Rev. A 72, 033818 (2005).

[31] P. Bertet, I. Chiorescu, G. Burkard, K. Semba, C.J.P. M. Harmans, D.P. DiVincenzo, and J.E. Mooij, Phys. Rev. Lett. 95, 257002 (2005).

[32] Y.X. Liu, L.F. Wei, and F. Nori, Phys. Rev. B 72, 014547 (2005).

[33] M. Steffen, M. Ansmann, R.C. Bialczak, N. Katz, E. Lucero, R. McDermott, M. Neeley, E.M. Weig, A.N. Cleland, and J.M. Martinis, Science 313, 1423 (2006).

[34] A. Maassen van den Brink, Phys. Rev. B 71, 064503 (2005). 